\title{
Modular representations of finite groups with trivial restriction to Sylow subgroups
}

Received January 2, 2012 and in revised form April 24, 2012

\begin{abstract}
Let $\mathbb{k}$ be a field of characteristic $p$. Let $G$ be a finite group of order divisible by $p$ and $P$ a $p$-Sylow subgroup of $G$. We describe the kernel of the restriction homomorphism $T(G) \rightarrow T(P)$, for $T(-)$ the group of endotrivial representations. Our description involves functions $G \rightarrow \mathbb{k}^{\times}$that we call weak $P$-homomorphisms. These are generalizations to possibly non-normal $P \leq G$ of the classical homomorphisms $G / P \rightarrow \mathbb{k}^{\times}$appearing in the normal case.
\end{abstract}

Keywords. Endotrivial module, trivial restriction to Sylow, weak homomorphism

\section{Introduction}

Let $\mathbb{k}$ be a field of characteristic $p>0$, not necessarily algebraically closed. Let $G$ be a finite group of order divisible by $p$. Although we are chiefly interested in the restriction to the $p$-Sylow subgroup, we can equally well describe the case of any subgroup $H \leq G$ whose index $[G: H]$ is invertible in $\mathbb{k}$, i.e. such that $H$ contains a $p$-Sylow subgroup of $G$. Consider the kernel of restriction

$$
T(G, H):=\operatorname{Ker}\left(\operatorname{Res}_{H}^{G}: T(G) \rightarrow T(H)\right),
$$

where we denote by $T(G)=T_{\mathbb{k}}(G)$ the abelian group of endotrivial $\mathbb{k} G$-modules. (See Remark 3.2.) Equivalently, $T(G, H)$ is the group of stable isomorphism classes of those $\mathbb{k} G$-modules whose restriction to $H$ is isomorphic to the trivial representation $\mathbb{k}$, up to projective summands (for such modules are necessarily endotrivial).

Endotrivial modules $M$ are important for various reasons, the most obvious one being that, by definition, the functor $M \otimes-$ provides an auto-equivalence on the stable category $\mathbb{k} G$-stab $=\mathbb{k} G$-mod $/ \mathbb{k} G$-proj. But there are further reasons to study them, as well as the larger class of so-called endopermutation modules, for instance as Green sources of simple modules. We refer the reader to Thévenaz [16] for a survey and more motivation. Let us simply indicate that the study of such modules has played a major role in the development of modular representation theory over the last decades. To borrow Alperin's words [1], a "triumph in finite group theory" has been their complete classification over

P. Balmer: Mathematics Department, UCLA, Los Angeles, CA 90095-1555, USA; e-mail: balmer@math.ucla.edu, URL: http://www.math.ucla.edu/ balmer 
p-groups, by work of Carlson-Thévenaz [7, 8] for endotrivial modules and Bouc [2] for endopermutation modules.

With this triumphant classification in mind, the natural question for a general group $G$ becomes to compare $T(G)$ to $T(P)$ for a $p$-Sylow subgroup $P \leq G$. This explains the importance of the kernel $T(G, P)$ in general. An extensive literature has recently flourished around this question (see for instance $[3,4,5,6,11,12,13,15]$ ), usually with the objective of describing $T(G, P)$ for specific classes of groups in very explicit terms (e.g. by generators and relations).

The goal of the present paper is to give a description of this kernel $T(G, H)$, valid for all $G$ and $H$, in purely elementary terms, notably not using stable categories, nor representations, but essentially only the action of $G$ by conjugation on the lattice of its p-subgroups.

In case the subgroup $H \triangleleft G$ is normal, it is well-known that $T(G, H)$ amounts to one-dimensional representations of the quotient $G / H$, that is, to group homomorphisms $G \rightarrow \mathbb{k}^{\times}$which are trivial on $H$. (Note that these coincide with all group homomorphisms $G \rightarrow \mathbb{k}^{\times}$if $H$ is the Sylow subgroup, since $\mathbb{k}$ has no nontrivial $p^{\text {th }}$ root of unity.) Our description of $T(G, H)$ for arbitrary, not necessarily normal $H \leq G$ involves a generalization of these homomorphisms, which we call "weak $H$-homomorphisms" from $G$ to $\mathbb{k}^{\times}$(Definition 2.2). These are functions $u: G \rightarrow \mathbb{k}^{\times}$which are constant on left and right $H$-cosets, which are trivial on $H$, and almost behave like group homomorphisms but not entirely. In fact, the relation $u\left(g_{2} g_{1}\right)=u\left(g_{2}\right) u\left(g_{1}\right)$ only holds for some pairs $g_{1}, g_{2}$ of elements of $G$. The deep reason why some of those relations are "lost" is that the stable category of the corresponding subgroup $H \cap H^{g_{1}} \cap H^{g_{2} g_{1}}$ vanishes. This happens exactly when that subgroup has order prime to the characteristic $p$. We come back to this phenomenon in Remark 4.11.

We shall construct explicit isomorphisms, in both directions, between the kernel $T(G, H)$ and the group $A(G, H)$ of weak $H$-homomorphisms $G \rightarrow \mathbb{k}^{\times}$.

It is high time we should give some precise definitions.

Beyond this introduction, the paper is organized as follows. In Section 2, we introduce weak $H$-homomorphisms and state the main theorems. These results are proved in Section 4 after recalling some basic modular representation theory in Section 3. The final Section 5 gives a couple of little corollaries of our description of $T(G, H)$, e.g. about the possible orders of elements in that finite abelian group.

\section{Weak $H$-homomorphisms and $T(G, H)$}

Fix $H \leq G$ a subgroup of index prime to $p$, for instance a $p$-Sylow subgroup. The following simple definition will be important throughout the paper.

2.1. Definition. We say that an element $g \in G$ is $H$-secant if the order $\left|H \cap H^{g}\right|$ is divisible by $p$, where of course $H^{g}=g^{-1} H g$ is the conjugate of $H$. In case $H \leq G$ is a p-Sylow, an element $g \in G$ is $H$-secant if and only if $H \cap H^{g}$ is non-trivial.

2.2. Definition. Define a weak $H$-homomorphism from $G$ to $\mathbb{k}^{\times}$to be a function $u$ : $G \rightarrow \mathbb{k}^{\times}$satisfying the following three properties: 
(WH1) For every $h \in H$, we have $u(h)=1$.

(WH2) For every non- $H$-secant $g$ (i.e. $\left|H \cap H^{g}\right|$ prime to $p$ ), we have $u(g)=1$.

(WH3) For every $g_{1}, g_{2} \in G$ such that $\left|H \cap H^{g_{1}} \cap H^{g_{2} g_{1}}\right|$ is divisible by $p$, we have

$$
u\left(g_{2} g_{1}\right)=u\left(g_{2}\right) \cdot u\left(g_{1}\right) .
$$

We define $A(G, H)$ to be the abelian group of weak $H$-homomorphisms from $G$ to $\mathbb{k}^{\times}$, under elementwise multiplication: $(u \cdot v)(g)=u(g) \cdot v(g)$ for every $g \in G$.

2.3. Examples. Here are two extreme cases where $T(G, H)$ is already well-known.

(1) Suppose that $H \triangleleft G$ is normal. Then every $g \in G$ is $H$-secant and every pair $g_{1}, g_{2} \in G$ satisfies (WH3). Hence $A(G, H)$ is the group $\operatorname{Hom}\left(G / H, \mathbb{k}^{\times}\right)$of group homomorphisms from the quotient $G / H$ to $\mathbb{k}^{\times}$.

(2) Suppose that $H \leq G$ is "strongly $p$-embedded", meaning that for every $g \in G$ not in $H$ the subgroup $H \cap H^{g}$ has order prime to $p$. Then $A(G, H)=1$ since (WH1) and (WH2) cover all possible $g \in G$ and force $u(g)=1$ everywhere.

Interestingly, the same group $A(G, H)$ is isomorphic to $T(G, H)$ in general, not only in those special cases. Let us explain how weak $H$-homomorphisms naturally appear in our problem. For this, it is convenient to use the following notation.

2.4. Remark. Let $g \in G$ and let $L$ and $K$ be subgroups of $G$ such that ${ }^{g} L \leq K$. We can combine twisting the action and restriction to a subgroup to obtain a $g$-twisted restriction functor ${ }^{g} \operatorname{Res}_{L}^{K}: \mathbb{k} K$-mod $\rightarrow \mathbb{k} L$-mod. It is defined by ${ }^{g} \operatorname{Res} M=M$ as a $\mathbb{k}$-vector space but with $L$ acting via $\ell \cdot m:=\left({ }^{g} \ell\right) m$ for all $\ell \in L$. On morphisms, ${ }^{g} \operatorname{Res}_{L}^{K}(f)=f$ as usual. It induces a functor ${ }^{g} \operatorname{Res}_{L}^{K}: \mathbb{k} K$-stab $\rightarrow \mathbb{k} L$-stab on stable categories.

Here is a first relation between $A(G, H)$ and endotrivial $\mathbb{k} G$-modules.

2.5. Construction. Let $M$ be an endotrivial $\mathbb{k} G$-module such that $\operatorname{Res}_{H}^{G} M \simeq \mathbb{k}$ in the stable category $\mathbb{k} H$-stab, i.e. the isomorphism class $[M]_{\simeq}$ belongs to our kernel $T(G, H)$. Choose an isomorphism $\xi: \mathbb{k} \stackrel{\sim}{\rightarrow} \operatorname{Res}_{H}^{G} M$ in $\mathbb{k} H$-stab. Then, for every $H$-secant element $g \in G$, consider the following subgroup of $H$ :

$$
H(g):=H \cap H^{g},
$$

whose order is divisible by $p$ by the assumption that $g$ is $H$-secant. Consider the two restrictions of $M$ to $H(g)$, namely the plain one $\operatorname{Res}_{H(g)}^{G} M$ and the $g$-twisted one ${ }^{g} \operatorname{Res}_{H(g)}^{G} M$ as in Remark 2.4. Note that $m \mapsto g m$ gives an $H(g)$-linear isomorphism $\operatorname{Res}_{H(g)}^{G} M \stackrel{\sim}{\rightarrow}{ }^{g} \operatorname{Res}_{H(g)}^{G} M$, simply denoted " $g$.". Note also that ${ }^{g}(H(g)) \leq H$ and that ${ }^{g} \operatorname{Res}_{H(g)}^{H} \mathbb{k}=\operatorname{Res}_{H(g)}^{H} \mathbb{k}=\mathbb{k}$. Note finally that the group of automorphisms of $\mathbb{k}$ in $\mathbb{k} H(g)$-stab is exactly $\mathbb{k}^{\times}$, via multiplication. This is where we use the assumption that $g$ is $H$-secant. Otherwise the stable category $\mathbb{k} H(g)$-stab would be trivial. So, there exists 
a unique scalar, that we call $u(g) \in \mathbb{k}^{\times}$, which makes the following diagram commute in $\mathbb{k} H(g)$-stab:

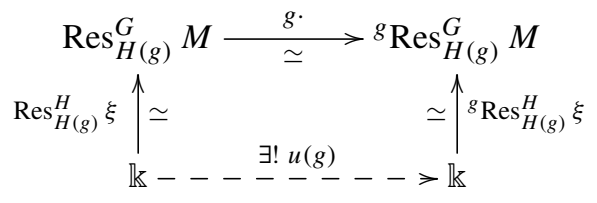

We shall see that the scalar $u(g)$ does not depend on the choice of $\xi$, nor on the choice of $M$ in its isomorphism class. Extending $u$ to non- $H$-secant $g$ by setting $u(g)=1$, we shall see that $u: G \rightarrow \mathbb{k}^{\times}$is a weak $H$-homomorphism in the sense of Definition 2.2. We denote this weak $H$-homomorphism $u$ by $v(M)$.

This construction actually gives us everything:

2.8. Theorem. Construction 2.5 induces a well-defined isomorphism

$$
v: \operatorname{Ker}(T(G) \rightarrow T(H)) \stackrel{\sim}{\rightarrow} A(G, H) .
$$

The proof is given in Section 4. This first construction explains how weak $H$-homomorphisms enter the picture. Let us now give the announced homomorphism $A(G, H) \rightarrow$ $T(G)$ more concretely. That is, let us describe what is the endotrivial module corresponding to a weak $H$-homomorphism. This will yield an inverse to $v$.

For $g \in G$, we denote by $[g]$ the class $g H$ of $g$ in the quotient $G / H$. We shall see that every weak $H$-homomorphism $u: G \rightarrow \mathbb{k}^{\times}$is constant on $H$-classes, hence $u([g]):=u(g)$ is well-defined on $G / H$. See Remark 4.2(2).

2.9. Theorem. Consider the $\mathbb{k} G$-module $\mathbb{k}(G / H)$ with usual left $G$-action on its $\mathbb{k}$-basis $G / H$. Let $u: G \rightarrow \mathbb{k}^{\times}$be a weak H-homomorphism (Definition 2.2). Define a map $e_{u}: \mathbb{k}(G / H) \rightarrow \mathbb{k}(G / H)$, depending on $u$, by the formula

$$
e_{u}([g])=\frac{1}{[G: H]} \sum_{d \in G / H} u(d)^{-1} \cdot g \cdot d
$$

for every $[g] \in G / H$, extended $\mathbb{k}$-linearly as always. Then we have:

(i) The homomorphism $e_{u}: \mathbb{k}(G / H) \rightarrow \mathbb{k}(G / H)$ is well-defined and $\mathbb{k} G$-linear. Moreover, it is an idempotent, $e_{u} \circ e_{u}=e_{u}$, in the stable category $\mathbb{k} G$-stab.

(ii) Since the category $\mathbb{k} G$-stab is idempotent complete (Remark 3.1), there exists a unique decomposition $\mathbb{k}(G / H) \cong M_{u} \oplus N_{u}$ in $\mathbb{k} G$-stab such that $e_{u}$ is the projection on $M_{u}$ along $N_{u}$; in other words, $e_{u}$ becomes $\left(\begin{array}{ll}1 & 0 \\ 0 & 0\end{array}\right)$ on $M_{u} \oplus N_{u}$.

(iii) The object $M_{u}$ is endotrivial and its restriction to $H$ is trivial.

(iv) The construction $u \mapsto\left[M_{u}\right]_{\simeq}$ described above yields a well-defined group homomorphism $A(G, H) \rightarrow T(G)$ which gives an isomorphism

$$
\alpha: A(G, H) \stackrel{\sim}{\rightarrow} \operatorname{Ker}(T(G) \rightarrow T(H)),
$$

inverse to the isomorphism $v$ of Theorem 2.8 .

This theorem is proven simultaneously with Theorem 2.8, in Section 4. 
2.11. Remark. We can try to reduce the amount of information involved in describing a weak $H$-homomorphism $u: G \rightarrow \mathbb{k}^{\times}$. Here is an alternative formulation which might be interesting for subgroups $H \leq G$ with small double quotient $H \backslash G / H$. First note that $g \in G$ being $H$-secant is a well-defined property of the class of $g$ in $G / H$ or even in $H \backslash G / H$. Of course, we call such classes $H$-secant as well. We already mentioned that $u \in A(G, H)$ is constant on left and right $H$-cosets (see Remark 4.2). It follows that we could describe weak $H$-homomorphisms $u \in A(G, H)$ as functions $u: H \backslash G / H \rightarrow \mathbb{k}^{\times}$ such that $u(H)=1, u(c)=1$ if $c$ is not $H$-secant and $u\left(c_{3}\right)=u\left(c_{2}\right) \cdot u\left(c_{1}\right)$ each time $c_{1}$, $c_{2}$ and $c_{3}$ are the classes of some elements $g_{1}, g_{2}$ and $g_{2} g_{1}$ for which $H \cap H^{g_{1}} \cap H^{g_{2} g_{1}}$ has order divisible by $p$. The latter condition, however, seems to depend on the choice of $g_{i} \in c_{i}$ for $i=1,2$. This is why we prefer formulation (WH3), to avoid confusion.

\section{Basics}

We recall some standard facts about modular representation theory of finite groups and fix some notation. In this section, $H \leq G$ can be any subgroup, not necessarily of index prime to $p$.

We denote by $\mathbb{k} G$-mod the category of finitely generated left $\mathbb{k} G$-modules and by $\mathbb{k} G$-stab $=\mathbb{k} G$-mod $/ \mathbb{k} G$-proj the stable category obtained as the additive quotient of the Frobenius abelian category $\mathbb{k} G$-mod by its subcategory of projective (=injective) modules. See Happel [10] for details. It is a triangulated category but, sadly enough, we shall not use this fact in this paper. The usual tensor product of representations, $M \otimes N=$ $M \otimes_{\mathbb{k}} N$ with diagonal $G$-action, passes to the stable category $\mathbb{k} G$-stab.

3.1. Remark. An additive category is idempotent complete (also known as karoubian or pseudo-abelian) if every idempotent endomorphism $e=e^{2}: A \rightarrow A$ yields a decomposition $A=\operatorname{im}(e) \oplus \operatorname{ker}(e)$, that is, a decomposition under which $e$ becomes $\left(\begin{array}{ll}1 & 0 \\ 0 & 0\end{array}\right)$. Such a decomposition is unique up to unique isomorphism.

The stable category $\mathbb{k} G$-stab is idempotent complete. This fact is folklore, e.g. since $\mathbb{k} G$-stab coincides with the thick subcategory of compact objects in the big stable category $\mathbb{k} G$-Stab of all, not necessarily finitely generated, $\mathbb{k} G$-modules modulo projectives and since $\mathbb{k} G$-Stab has infinite coproducts, hence is idempotent complete (use [14, Prop. 1.6.8]).

Alternatively, one can sketch a direct proof as follows. Let $M$ be a finitely generated $\mathbb{k} G$-module that we can assume without projective summand. The latter assumption implies that any endomorphism of $M$ which vanishes in $\mathbb{k} G$-stab is nilpotent (using nilpotence of the Jacobson radical). Hence, if $e: M \rightarrow M$ is an endomorphism in $\mathbb{k} G$-mod such that $e^{2}=e$ in $\mathbb{k} G$-stab, then $h=e^{2}-e$ is nilpotent in $\mathbb{k} G$-mod. The usual lifting of idempotents modulo nilpotents yields a correction $\tilde{e}$ of $e$ such that $\tilde{e}^{2}=\tilde{e}$ in $\mathbb{k} G$-mod already, with the same $\tilde{e}=e$ in $\mathbb{k} G$-stab. (By induction, reduce to the case $h^{2}=0$ and verify that $\tilde{e}=e+h-2 e h$ will do in that case; note that $e h=h e$.) Then we have $M=\operatorname{im}(\tilde{e}) \oplus \operatorname{ker}(\tilde{e})$ in $\mathbb{k} G$-mod, inducing the wanted decomposition in $\mathbb{k} G$-stab.

3.2. Remark. Recall that a $\mathbb{k} G$-module $M$ is endotrivial if $M$ is $\otimes$-invertible $M \otimes M^{*} \simeq \mathbb{1}$ in the stable category $\mathbb{k} G$-stab, or, in eponymic terms, if its module of endomorphisms 
is the trivial $\mathbb{k} G$-module, up to projective: $\operatorname{End}_{\mathbb{k}}(M) \simeq \mathbb{k} \oplus$ (proj). Being endotrivial can be tested on the elementary abelian $p$-subgroups of $G$ by Chouinard's Theorem [9]. In particular, it suffices that $\operatorname{Res}_{P}^{G} M$ be endotrivial on the $p$-Sylow $P \leq G$ or any subgroup in between, $P \leq H \leq G$. (See also Remark 3.4.)

3.3. Notation. For a subgroup $H \leq G$, we denote the class $g H \in G / H$ by $[g]_{H}$ or just $[g]$ when $H$ is obvious from the context. Here, $\mathbb{k}(G / H)$ will always be a left $\mathbb{k} G$-module via $g \cdot[x]=[g x]$. We have the usual restriction-induction adjunction

$$
\operatorname{Res}_{H}^{G}: \mathbb{k} G \text {-stab } \leftrightarrows \mathbb{k} H \text {-stab : } \operatorname{Ind}_{H}^{G}=\mathbb{k} G \otimes_{\mathbb{k} H}-
$$

whose unit $\eta_{M}: M \rightarrow \mathbb{k} G \otimes_{\mathbb{k} H} M$ is given by $m \mapsto \sum_{[g] \in G / H} g \otimes g^{-1} m$.

3.4. Remark. Assume that a subgroup $H \leq G$ has index $[G: H]$ prime to $p$. Then the above unit $\eta:$ Id $\rightarrow \operatorname{Ind}_{H}^{G} \operatorname{Res}_{H}^{G}$ has a retraction $\pi: \operatorname{Ind}_{H}^{G} \operatorname{Res}_{H}^{G} \rightarrow$ Id, namely $\pi_{M}: \mathbb{k} G \otimes_{\mathbb{k} H} M \rightarrow M$ defined by $g \otimes m \mapsto \frac{1}{[G: H]} g m$. It follows that the functor $\operatorname{Res}_{H}^{G}: \mathbb{k} G$-stab $\rightarrow \mathbb{k} H$-stab is faithful. Indeed, if $\operatorname{Res}(f)=0$ then $f=\pi \circ \eta \circ f=$ $\pi \circ$ Ind $\operatorname{Res}(f) \circ \eta=0$ by naturality of $\eta$. (Of course, Res is usually not full.) Then $\operatorname{Res}_{H}^{G}: \mathbb{k} G$-stab $\rightarrow \mathbb{k} H$-stab detects vanishing of objects. Applying this property with the cone (cokernel) of the obvious morphism $\mathbb{k} \rightarrow \operatorname{End}_{\mathbb{k}}(M) \cong M \otimes M^{*}$, we see that if $\operatorname{Res}_{H}^{G} M$ is $\otimes$-invertible then so is $M$. Hence the functor $\operatorname{Res}_{H}^{G}$ detects endotriviality, as already mentioned. More generally, applying faithfulness to the cone of any morphism in $\mathbb{k} G$-stab shows that $\operatorname{Res}_{H}^{G}$ detects isomorphisms.

3.5. Remark (Mackey formulas). Let $K, L \leq H$ be two subgroups of the same group $H$. We shall use a couple of Mackey bijections between some left $K$-sets and some Mackey isomorphisms between left $\mathbb{k} K$-modules. We therefore recall them together beforehand. Let $T \subset H$ be a set of representatives of $K \backslash H / L$. For every $t \in T$, consider the morphism of left $H$-sets

$$
\operatorname{mack}_{t}: H /{ }^{t} L \rightarrow H / L \quad \text { defined by } \operatorname{mack}_{t}\left([h]_{t}\right)=[h t]_{L} .
$$

One instance of the Mackey formula tells us that the map obtained by restricting these maps to the subsets $K /\left(K \cap{ }^{t} L\right)$ of $H /{ }^{t} L$ and taking their coproduct over $t \in T$ yields a bijection of left $K$-sets:

$$
\text { mack : } \coprod_{t \in T} K /\left(K \cap{ }^{t} L\right) \stackrel{\sim}{\rightarrow} H / L, \quad[k]_{K \cap{ }^{t} L} \mapsto \operatorname{mack}_{t}([k])=[k t]_{L} .
$$

Similarly, for every $\mathbb{k} L$-module $N$, there is a Mackey isomorphism of $\mathbb{k} K$-modules, $\bigoplus_{t \in T} \operatorname{Ind}_{K \cap L}^{K}{ }^{t}{ }^{-1} \operatorname{Res}_{K \cap t}^{L} N \stackrel{\sim}{\rightarrow} \operatorname{Res}_{K}^{H} \operatorname{Ind}_{L}^{H} N$, still denoted mack and given by

$$
\text { mack }: \bigoplus_{t \in T} \mathbb{k} K \otimes_{\mathbb{k}\left(K \cap^{t} L\right)} N \stackrel{\simeq}{\rightarrow} \mathbb{k} H \otimes_{\mathbb{k} L} N, \quad x \otimes y \mapsto x t \otimes y .
$$

Recall from Remark 2.4 that by definition of the twisted restriction ${ }^{t-1} \operatorname{Res}_{K \cap t}^{L} \cap^{t} N$, each subgroup $K \cap{ }^{t} L$ acts on the corresponding factor $N$ appearing on the left-hand side of (3.7) via $k \cdot n=k^{t} n$, observing that $k^{t} \in L$ for every $k \in K \cap{ }^{t} L$. 
Let $H$ be a finite group, let $X$ be a left $H$-set and let $x \in X$. Then we denote by $H_{x}=\{h \in H \mid h x=x\}$ the stabilizer of $x$ in $H$. The following result will be essential for our computations in stable categories.

3.8. Lemma. Let $H$ be a finite group and let $X, Y$ be two finite left $H$-sets. Let $f: \mathbb{k} X \rightarrow$ $\mathbb{k} Y$ be a $\mathbb{k} H$-linear homomorphism. It is given by scalars $a_{x, y} \in \mathbb{k}$ indexed by $x \in X$ and $y \in Y$ such that $f(x)=\sum_{y \in Y} a_{x, y} y$ for every $x \in X$.

If $a_{x, y}=0$ for every $x \in X$ and $y \in Y$ such that $p$ divides $\left|H_{x} \cap H_{y}\right|$ then the morphism $f$ is zero in $\mathbb{k} H$-stab, i.e. it factors via a projective $\mathbb{k} H$-module.

Proof. The $\mathbb{k} H$-linearity of $f$ gives us for every $h \in H, x \in X$ and $y \in Y$ that

$$
a_{h x, h y}=a_{x, y} .
$$

Now, consider the diagonal action of $H$ on $X \times Y$ and note that the property that $\left|H_{x} \cap H_{y}\right|$ is prime to $p$ is constant on the $H$-orbit of $(x, y) \in X \times Y$. Let $S \subset X \times Y$ be a set of representatives of only those $H$-orbits in $X \times Y$ on which $\left|H_{x} \cap H_{y}\right|$ is prime to $p$. Consider the free $\mathbb{k} H$-module $\operatorname{Ind}_{1}^{H} \operatorname{Res}_{1}^{H}(\mathbb{k} Y)=\mathbb{k} H \otimes_{k} \mathbb{k} Y$ (that is, with $H$-action only on the left factor) and the morphisms $f_{1}$ and $f_{2}$ as follows:

$$
\sum_{h \in H} \sum_{\substack { x \\
\begin{subarray}{c}{\left.x_{0}, y_{0}\right) \in S \\
x=h x_{0}{ x \\
\begin{subarray} { c } { x _ { 0 } , y _ { 0 } ) \in S \\
x = h x _ { 0 } } }\end{subarray}}\left|H_{x_{0}} \cap{ }^{\downarrow} H_{y_{0}}\right|^{-1} a_{x_{0}, y_{0}} \cdot h \otimes y_{0}
$$
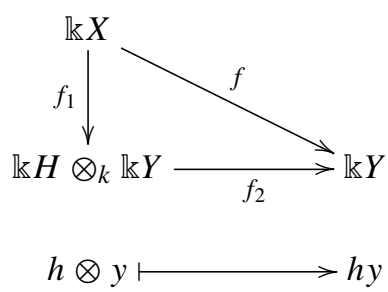

The $\mathbb{k} H$-linearity of $f_{2}$ is immediate and that of $f_{1}$ is easy by a standard change of variables on the summation index $h \in H$. It now suffices to check that $f_{2} \circ f_{1}=f$. Let $x \in X$ and let $b_{x, y} \in \mathbb{k}$ for all $y \in Y$ be such that $f_{2} \circ f_{1}(x)=\sum_{y \in Y} b_{x, y} y$. By the above construction, we have

$$
b_{x, y}=\sum_{h \in H} \sum_{\substack{\left(x_{0}, y_{0}\right) \in S \\ x=h x_{0} \text { and } y=h y_{0}}}\left|H_{x_{0}} \cap H_{y_{0}}\right|^{-1} a_{x_{0}, y_{0}} .
$$

We want to prove that $b_{x, y}=a_{x, y}$ for all $y \in Y$. If $p$ divides $\left|H_{x} \cap H_{y}\right|$, there is no $\left(x_{0}, y_{0}\right) \in S$ with $(x, y)=\left(h x_{0}, h y_{0}\right)$ by choice of $S$, hence the above summation is empty in that case and we get $b_{x, y}=0$ which coincides with $a_{x, y}$ by hypothesis. Now, suppose that $\left|H_{x} \cap H_{y}\right|$ is prime to $p$, then the index $\left(x_{0}, y_{0}\right) \in S$ of the above sum is unique. However, there are many $h \in H$ with the property that $\left(h x_{0}, h y_{0}\right)=(x, y)$, namely there are $\left|H_{x_{0}} \cap H_{y_{0}}\right|$ of them. In that case, we obtain $b_{x, y}=a_{x_{0}, y_{0}}$ but the latter is also equal to $a_{x, y}$ by (3.9) since $(x, y)=\left(h x_{0}, h y_{0}\right)$. 


\section{Proof of the theorems}

As in Section $2, H \leq G$ is a subgroup of index prime to $p$. Let us denote this index by $n:=[G: H]$ for short. So, $1 / n$ exists in our field $\mathbb{k}$. Recall that $g \in G$ is $H$-secant if $p$ divides the order of $H(g):=H \cap H^{g}$.

It might be reassuring to start with the following.

4.1. Example. Let $u \equiv 1$ be the trivial weak $H$-homomorphism from $G$ to $\mathbb{k}^{\times}$. Then, under the identification $\mathbb{k}(G / H) \cong \operatorname{Ind}_{H}^{G} \operatorname{Res}_{H}^{G} \mathbb{k}=\mathbb{k} G \otimes_{\mathbb{k} H} \mathbb{k}$, given by $[g] \mapsto g \otimes 1$, the endomorphism $e_{u}$ of $\mathbb{k}(G / H)$ (see (2.10)) coincides with the idempotent $\eta_{\mathbb{k}} \circ \pi_{\mathbb{k}}$ of Ind Res $\mathbb{k}$ (see Remark 3.4). Indeed, for every $g \in G$, we have

$$
e_{1}([g])=\frac{1}{n} \sum_{d \in G / H} g d=\frac{1}{n} \sum_{d^{\prime} \in G / H} d^{\prime}=\eta_{\mathbb{k}}\left(\frac{1}{n}\right)=\eta_{\mathbb{k}}\left(\pi_{\mathbb{k}}([g])\right) .
$$

So this idempotent corresponds to the trivial module $\mathbb{k}$ appearing as a direct summand of $\mathbb{k}(G / H)$ via $\eta_{\mathbb{k}}$.

4.2. Remarks. Let $u: G \rightarrow \mathbb{k}^{\times}$be a weak $H$-homomorphism (Definition 2.2). We shall repeatedly use the following facts, often without mention.

(1) We have $u\left(g^{-1}\right)=u(g)^{-1}$ for every $g \in G$. Indeed, by (WH2), we can assume that $g$, or equivalently $g^{-1}$, is $H$-secant. Then $1=u(1)=u\left(g^{-1}\right) u(g)$ follows by (WH1) and (WH3) since $p$ divides $\left|H \cap H^{g} \cap H^{1}\right|$.

(2) For every $g \in G$ and $h \in H$, we have $u(h g)=u(g)=u(g h)$. To see this, note that $g, h g$ and $g h$ are simultaneously $H$-secant. By (WH2), we can assume that they are all $H$-secant. In that case, the groups $H \cap H^{g} \cap H^{h g}=H \cap H^{g}$ and $H \cap H^{h} \cap H^{g h}=$ $H \cap H^{g h}$ have order divisible by $p$, and the relations follow from (WH3) and the fact that $u(h)=1$ by (WH1).

4.3. Proposition. Let $u: G \rightarrow \mathbb{k}^{\times}$be a weak $H$-homomorphism (Definition 2.2). Then the endomorphism $e_{u}: \mathbb{k}(G / H) \rightarrow \mathbb{k}(G / H)$ given in (2.10) is well-defined and $\mathbb{k} G$ linear.

Proof. Let $g \in G$ and consider the well-defined element

$$
e_{u}(g)=\frac{1}{n} \sum_{d \in G / H} u(d)^{-1} \cdot g \cdot d
$$

in $\mathbb{k}(G / H)$, as in (2.10). We want to show that $e_{u}(g)=e_{u}(g h)$ for every $h \in H$ :

$$
e_{u}(g h)=\frac{1}{n} \sum_{d \in G / H} u(d)^{-1} g h d=\frac{1}{n} \sum_{d^{\prime} \in G / H} u\left(d^{\prime}\right)^{-1} g d^{\prime}=e_{u}(g)
$$

using the change of variables $d^{\prime}=h d$ on $G / H$ which preserves the value of the scalar $u\left(d^{\prime}\right)=u(h d)=u(d)$ by Remark 4.2(2). Hence, $e_{u}(g)$ only depends on $[g]_{H} \in G / H$, which means that $e_{u}$ is well-defined. It is clearly $\mathbb{k} G$-linear by definition of the action of $G$ on $G / H$, which appears on the left of $g$ (and of course commutes with the scalar $\left.u(d)^{-1} \in \mathbb{k}\right)$. 
4.4. Main Lemma. Let $u \in A(G, H)$ and $\underline{u}: \operatorname{Res}_{H}^{G}(\mathbb{k}(G / H)) \rightarrow \mathbb{k}$ its $\mathbb{k}$-linear extension, i.e. mapping every basis element $c \in \bar{G} / H$ to $u(c)$. This $\underline{u}$ is $\mathbb{k} H$-linear. Consider the $\mathbb{k} H$-linear homomorphism $w: \mathbb{k} \rightarrow \operatorname{Res}_{H}^{G}(\mathbb{k}(G / H))$ given by

$$
w(1)=\frac{1}{n} \sum_{d \in G / H} u(d)^{-1} \cdot d
$$

Then $\underline{u} \circ w=\operatorname{id}_{\mathbb{k}}$ and $w \circ \underline{u}=\operatorname{Res}_{H}^{G}\left(e_{u}\right)$ in $\mathbb{k} H$-stab.

Proof. It is easy to verify that both $\underline{u}$ and $w$ are indeed $\mathbb{k} H$-linear (see Remark 4.2(2) if necessary). Let us also observe right away that $\underline{u} \circ w=\operatorname{id}_{\mathbb{k}}$ :

$$
\underline{u}(w(1))=\frac{1}{n} \sum_{d \in G / H} u(d)^{-1} \cdot u(d)=\frac{|G / H|}{n}=1 .
$$

So, let us prove that $w \circ \underline{u}=e_{u}$ in $\mathbb{k} H$-stab. We are going to use Lemma 3.8 for the left $H$-set $X=Y=G / H=\operatorname{Res}_{H}^{G}(G / H)$ and the morphism $f=w \circ \underline{u}-e_{u}: \mathbb{k} X \rightarrow \mathbb{k} Y$, which we claim is zero in $\mathbb{k} H$-stab. For every $x \in G / H$, we have

$$
w \circ \underline{u}(x)=\frac{1}{n} \sum_{y \in Y} u(x) u(y)^{-1} \cdot y .
$$

On the other hand, let us choose $g_{1} \in G$ such that $x=\left[g_{1}^{-1}\right]_{H}$. Then we have

$$
e_{u}(x)=\frac{1}{n} \sum_{d \in G / H} u(d)^{-1} \cdot g_{1}^{-1} \cdot d=\frac{1}{n} \sum_{y \in Y} u\left(g_{1} y\right)^{-1} \cdot y
$$

using the change of variables $d=g_{1} y$ on $Y=G / H$. By Lemma 3.8, in the above expressions for $w \circ \underline{u}(x)$ and $e_{u}(x)$, it suffices to identify the coefficients of only those $y \in Y$ such that $p$ divides $\left|H_{x} \cap H_{y}\right|$. But here the stabilizers are $H_{x}=H \cap{ }^{x} H$ and $H_{y}=H \cap{ }^{y} H$. So we can assume that $y \in Y$ is such that $p$ divides $\left|H \cap{ }^{x} H \cap{ }^{y} H\right|$. Choose $g_{2} \in G$ such that $\left[g_{2}^{-1}\right]_{H}=g_{1} y$. Note that then $\left[\left(g_{2} g_{1}\right)^{-1}\right]_{H}=y$. Since $p$ divides $\left|H \cap \cap^{x} H \cap{ }^{y} H\right|=\left|H \cap H^{g_{1}} \cap H^{g_{2} g_{1}}\right|$, property (WH3) of $u$ together with Remark 4.2 gives us

$$
u(y)^{-1}=u\left(g_{2} g_{1}\right)=u\left(g_{2}\right) u\left(g_{1}\right)=u\left(g_{1} y\right)^{-1} u(x)^{-1},
$$

hence the wanted $u(x) u(y)^{-1}=u\left(g_{1} y\right)^{-1}$.

4.6. Proposition. For every weak $H$-homomorphism $u: G \rightarrow \mathbb{k}^{\times}$, we have an idempotent $e_{u} \circ e_{u}=e_{u}$ on the object $\mathbb{k}(G / H)$ of $\mathbb{k} G$-stab. Moreover, the corresponding direct summand $M_{u}$ of $\mathbb{k}(G / H)$ restricts to $\mathbb{k}$ on $\mathbb{k} H$-stab, that is, $\operatorname{Res}_{H}^{G} M_{u} \simeq \mathbb{k}$. Finally, $M_{u}$ is an endotrivial $\mathbb{k} G$-module. 
Proof. Since the functor $\operatorname{Res}_{H}^{G}: \mathbb{k} G$-stab $\rightarrow \mathbb{k} H$-stab is faithful (Remark 3.4), it suffices to verify $\left(\operatorname{Res}\left(e_{u}\right)\right)^{2}=\operatorname{Res}\left(e_{u}\right)$ in $\mathbb{k} H$-stab, where it follows directly from our Main Lemma 4.4 since $\operatorname{Res}\left(e_{u}\right)=w \circ \underline{u}$ and $\underline{u} \circ w=\operatorname{id}_{\mathbb{k}}$ in $\mathbb{k} H$-stab:

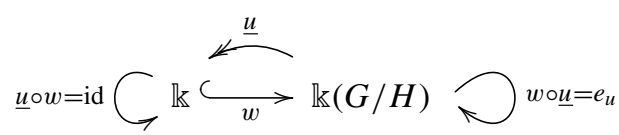

The direct summand of the object $\operatorname{Res}_{H}^{G}(\mathbb{k}(G / H))=\mathbb{k}(G / H)$ of $\mathbb{k} H$-stab corresponding to the idempotent $w \circ \underline{u}$ is simply $\mathbb{k}$. This proves the second claim (see Remark 3.1). Finally, $\operatorname{Res}_{H}^{G}$ being faithful, it detects endotriviality by Remark 3.4 (or Chouinard's Theorem [9]). So, $M_{u}$ is an endotrivial $\mathbb{k} G$-module.

4.7. Remark. Here is another approach to the idempotent $e_{u}$. By (WH2), the value $u(g)$ is only interesting when $g \in G$ is $H$-secant. Similarly, the only $g_{1}, g_{2}$ satisfying the hypothesis of (WH3) must be $H$-secant, and so must be $g_{2} g_{1}$ (easy exercise). Continuing in this vein, we can use Lemma 3.8 and Remark 3.4 to show that the endomorphism $e_{u}$ of $\mathbb{k}(G / H)$ is equal in $\mathbb{k} G$-stab to

$$
[g]_{H} \mapsto \frac{1}{[G: H]} \sum_{\substack{d \in G / H \\ H \text {-secant }}} u(d)^{-1} \cdot g \cdot d .
$$

This formula makes it apparent that only the values $u(d)$ for $H$-secant $d$ are relevant.

We now turn to the other side of the game, namely the construction of the homomorphism $v: T(G, H) \rightarrow A(G, H)$.

4.8. Remark. For $g \in G$ and subgroups $K, L \leq G$ such that ${ }^{g} L \leq K$, recall the twisted restriction ${ }^{g} \operatorname{Res}_{L}^{K}: \mathbb{k} K$-stab $\rightarrow \mathbb{k} L$-stab as in Remark 2.4. When ${ }^{g_{1}} L \leq K$ and ${ }^{g_{2}} K \leq H$ then ${ }^{g_{2} g_{1}} L \leq H$ and it is easy to check that we have an equality of functors ${ }^{g_{2} g_{1}} \operatorname{Res}_{L}^{H}=$ ${ }^{g_{1}} \operatorname{Res}_{L}^{K} \circ{ }^{g_{2}} \operatorname{Res}_{K}^{H}$. (Watch the order of the $g_{i}$ !) The latter equality will often be used tacitly in what follows.

Recall Construction 2.5, which associates a function $u: G \rightarrow \mathbb{k}^{\times}$to every $\mathbb{k} G$ module $M$ coming with an isomorphism $\xi: \mathbb{k} \stackrel{\sim}{\rightarrow} \operatorname{Res}_{H}^{G} M$ in $\mathbb{k} H$-stab. For convenience, we repeat diagram (2.7) whose commutativity defines $u(g)$ for every $H$-secant $g \in G$ (recall that $H(g)$ stands for $H \cap H^{g}$ ):

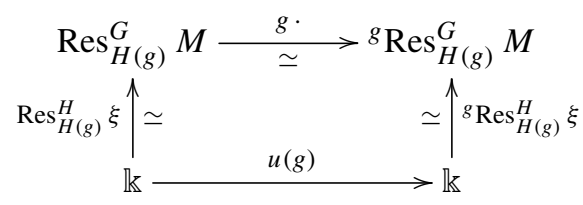

For non- $H$-secant $g \in G$, we defined $u(g)=1$. Note however that (4.9) also trivially commutes in that case since $\mathbb{k} H(g)$-stab $=0$ for non- $H$-secant $g$. 
4.10. Lemma. The scalar $u(g)$ as in (4.9) does not depend on the choice of the isomorphism $\xi$. Moreover, $u: G \rightarrow \mathbb{k}^{\times}$is a weak H-homomorphism.

Proof. For the first part, we can assume that $g$ is $H$-secant, for otherwise $u(g)=1$ anyway. Let $\xi^{\prime}: \mathbb{k} \stackrel{\sim}{\rightarrow} \operatorname{Res}_{H}^{G} M$ be another isomorphism and $u^{\prime}(g)$ the associated scalar, i.e. $u^{\prime}(g)=\left({ }^{g} \operatorname{Res}\left(\xi^{\prime}\right)\right)^{-1} \circ(g \cdot) \circ \operatorname{Res}\left(\xi^{\prime}\right)$. Then $\xi^{-1} \xi^{\prime}: \mathbb{k} \stackrel{\sim}{\rightarrow} \mathbb{k}$ is an automorphism of $\mathbb{k}$ in $\mathbb{k} H$-stab, hence it is given by an invertible scalar $v \in \mathbb{k}^{\times}$. So, we have $\xi^{\prime}=v \cdot \xi=\xi \cdot v$. Hence $v$ and $v^{-1}$ cancel out in $u^{\prime}(g)$, giving $u^{\prime}(g)=u(g)$.

Let us now check that $u$ is a weak $H$-homomorphism as in Definition 2.2. To check (WH1), it suffices to use that $\xi$ is $H$-linear and that $\mathbb{k}$ has trivial action. Indeed, for every $h \in H$ (necessarily $H$-secant) we have a commutative square

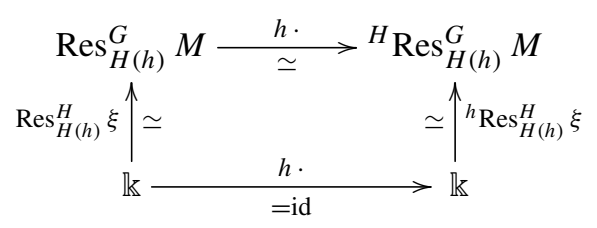

This proves $u(h)=1$. Property (WH2) holds by construction. Let us verify (WH3). Suppose that $g_{1}$ and $g_{2}$ are such that the subgroup $L:=H \cap H^{g_{1}} \cap H^{g_{2} g_{1}}$ has order divisible by $p$. This means that $\mathbb{k} L$-stab is not the zero category and endomorphisms of $\mathbb{k}$ in that category identify with $\mathbb{k}$ (under multiplication, as usual). Consider the following commutative diagram in $\mathbb{k} L$-stab:

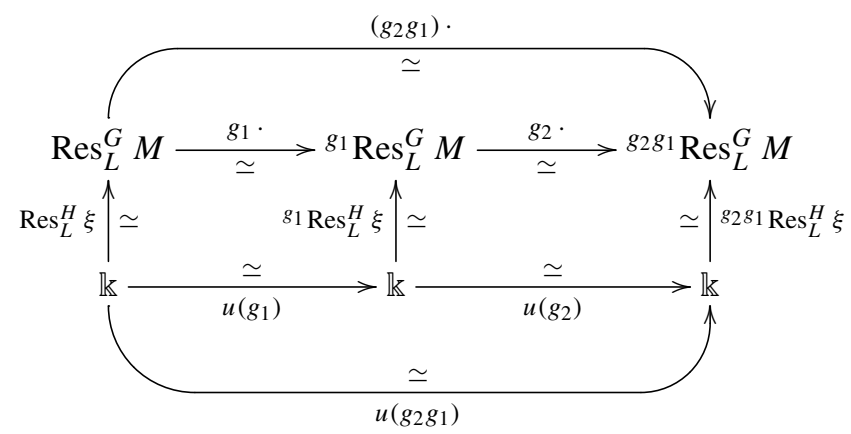

The left-hand central square commutes by (4.9) for $g_{1}$, restricted to $L$. The right-hand one commutes by (4.9) for $g_{2}$ after applying ${ }^{g_{1}} \operatorname{Res}_{L}{ }^{H\left(g_{2}\right)}$ to it, using that ${ }^{g_{1}} L \leq H \cap H^{g_{2}}=$ $H\left(g_{2}\right)$ and the relation ${ }^{g_{2} g_{1}} \operatorname{Res}_{L}^{H}={ }^{g_{1}} \operatorname{Res}_{L}^{H\left(g_{2}\right)}{ }^{\circ}{ }^{g_{2}} \operatorname{Res}_{H\left(g_{2}\right)}^{H}$ already seen in Remark 4.8. The outside square commutes by (4.9) again but now for $g_{2} g_{1}$. Hence the lower "triangle" gives us $u\left(g_{2} g_{1}\right)=u\left(g_{2}\right) u\left(g_{1}\right)$ as wanted.

4.11. Remark. In the above proof, it is essential that $L=H \cap H^{g_{1}} \cap H^{g_{2} g_{1}}$ has order

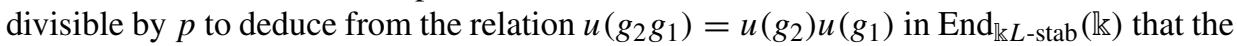
same relation holds in $\mathbb{k}$. When $L$ has order prime to $p$, this stable endomorphism ring is trivial and $\mathbb{k} \rightarrow \operatorname{End}_{\mathbb{k} L \text {-stab }}(\mathbb{k})$ is not injective. This is why the "homomorphism property 
of $u ", u\left(g_{2} g_{1}\right)=u\left(g_{2}\right) u\left(g_{1}\right)$, does not hold for general $g_{1}$ and $g_{2}$ and why we are left with weak homomorphisms as in Definition 2.2 (WH3).

4.12. Proposition. The assignment $M \mapsto u$ as in Construction 2.5 yields a well-defined group homomorphism $v: T(G, H) \rightarrow A(G, H)$.

Proof. Suppose that $M^{\prime}$ is isomorphic to $M$ in $\mathbb{k} G$-stab and let $u=v(M)$ and $u^{\prime}=$ $v\left(M^{\prime}\right)$. For $g$ non- $H$-secant, we have $u(g)=1=u^{\prime}(g)$ by definition. So, let $g \in G$ be $H$-secant. There exists a $\mathbb{k} G$-linear morphism $f: M \rightarrow M^{\prime}$ such that $f$ is an isomorphism in $\mathbb{k} G$-stab, hence also in $\mathbb{k} H$-stab after restriction. We can then create the following commutative cube in $\mathbb{k} H(g)$-stab:

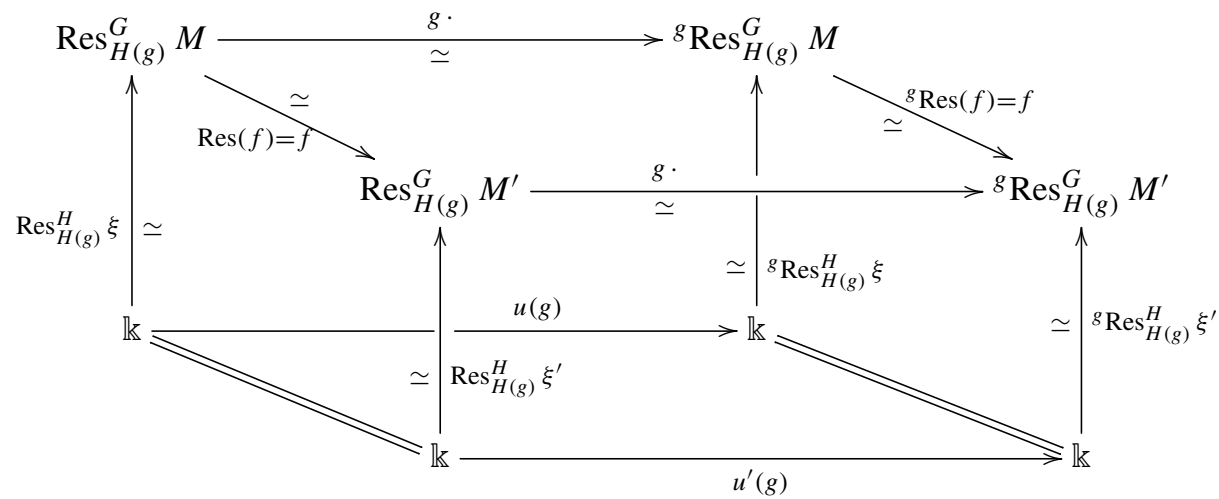

whose back and front squares are (4.9) for $M$ and $M^{\prime}$ respectively. To compute $u^{\prime}(g)$, we choose $\xi^{\prime}: \mathbb{k} \stackrel{\sim}{\rightarrow} \operatorname{Res}_{H}^{G} M^{\prime}$ in $\mathbb{k} H$-stab to be $\operatorname{Res}(f) \circ \xi$, which is allowed by Lemma 4.10. This makes the side squares commute. The top square commutes by $\mathbb{k} G$-linearity of $f$. Hence the bottom square commutes, which shows that $u$ is independent of the choice of $M$ in its isomorphism class in $\mathbb{k} G$-stab.

Finally, for $i=1,2$, let $M_{i}$ be a $\mathbb{k} G$-module, let $\xi_{i}: \mathbb{k} \stackrel{\sim}{\rightarrow} \operatorname{Res}_{H}^{G} M_{i}$ be an isomorphism in $\mathbb{k} H$-stab and let $u_{i}:=v\left(M_{i}\right)$. To compute $v\left(M_{1} \otimes M_{2}\right)$, we can use the isomorphism $\xi_{1} \otimes \xi_{2}: \mathbb{k} \stackrel{\sim}{\rightarrow} \operatorname{Res}_{H}^{G}\left(M_{1} \otimes M_{2}\right)$ in $\mathbb{k} H$-stab. Again, we can assume that $g \in G$ is $H$-secant. Tensoring the two commutative squares (4.9) defining $u_{1}(g)$ and $u_{2}(g)$, we get the following commutative diagram in $\mathbb{k} H(g)$-stab:

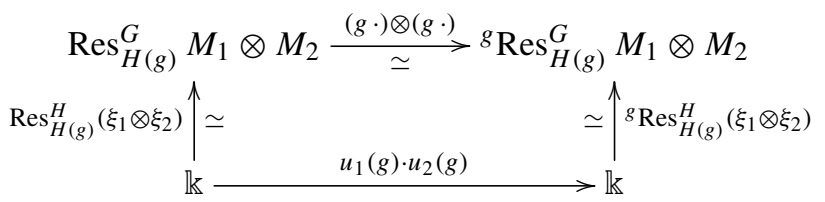

Now observe that the multiplication $g \cdot$ on the $\mathbb{k} G$-module $M_{1} \otimes M_{2} \rightarrow M_{1} \otimes M_{2}$ is precisely given by $m_{1} \otimes m_{2} \mapsto\left(g m_{1}\right) \otimes\left(g m_{2}\right)$. So, it coincides with the top morphism in the above square. Hence $u_{1}(g) \cdot u_{2}(g)$ must be equal to $v\left(M_{1} \otimes M_{2}\right)(g)$ by (4.9) again, but this time applied to $M_{1} \otimes M_{2}$. 
We now need a small result, which palliates the lack of multiplicativity of weak $\mathrm{H}$ homomorphisms.

4.13. Lemma. Let $u: G \rightarrow \mathbb{k}^{\times}$be a weak $H$-homomorphism. Let $g \in G$ be $H$-secant and let $s \in G$. Let us abbreviate $H(g, s):=H \cap H^{g} \cap{ }^{s} H$. Then, in $\mathbb{k}$,

$$
[H(g): H(g, s)] \cdot u(g s)=[H(g): H(g, s)] \cdot u(g) \cdot u(s) .
$$

Proof. The result is trivial if $p$ divides the number $[H(g): H(g, s)]$ which appears on both sides. So, we can assume that $p$ does not divide that index. But since $g$ is $H$-secant, $p$ divides $|H(g)|$. These two facts force $p$ to divide $|H(g, s)|=\left|H \cap H^{g} \cap H^{s^{-1}}\right|=$ $\left|H^{s} \cap H^{g s} \cap H\right|$. Hence, by (WH3), for $g_{2}=g$ and $g_{1}=s$, we have $u(g s)=u(g) \cdot u(s)$ and the result also holds in that case.

4.14. Proposition. Let $u: G \rightarrow \mathbb{k}^{\times}$be a weak $H$-homomorphism and $M_{u}$ the associated endotrivial $\mathbb{k} G$-module, as in Proposition 4.6. Then $v\left(M_{u}\right)=u$.

Proof. By construction of $M_{u}$, there exist a $\mathbb{k} G$-module $N_{u}$ and a $\mathbb{k} G$-linear homomorphism $f: M_{u} \oplus N_{u} \rightarrow \mathbb{k}(G / H)$ which is an isomorphism in $\mathbb{k} G$-stab and such that the idempotent $e_{u}$ on $\mathbb{k}(G / H)$ becomes $\left(\begin{array}{cc}1 & 0 \\ 0 & 0\end{array}\right)$ on $M_{u} \oplus N_{u}$, again in $\mathbb{k} G$-stab. After restriction to $H$, we also know that the idempotent $e_{u}$ corresponds to the direct summand $\mathbb{k}$ embedding in $\operatorname{Res}(\mathbb{k}(G / H))$ via $w: \mathbb{k} \rightarrow \mathbb{k}(G / H)$, as in (4.5). Since such a decomposition is unique (Remark 3.1), we can choose an isomorphism $\xi: \mathbb{k} \stackrel{\sim}{\rightarrow} \operatorname{Res}_{H}^{G} M_{u}$ in $\mathbb{k} H$-stab such that the following diagram commutes:

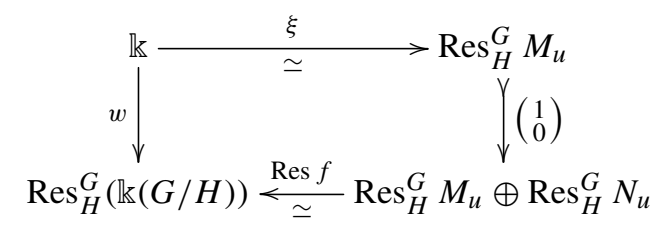

in $\mathbb{k} H$-stab. For every $H$-secant $g \in G$, consider the following commutative diagram in $\mathbb{k} H(g)$-stab:

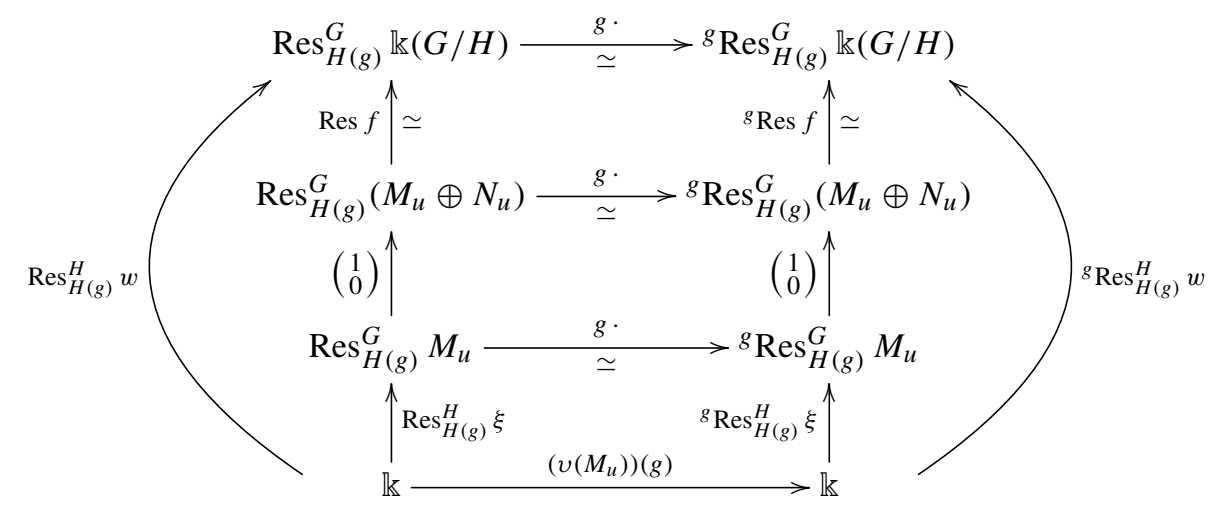


where the unit $\left(v\left(M_{u}\right)\right)(g)$ at the bottom is the one of Construction 2.5 associated to $M_{u}$, which makes the lower square commute. The middle and upper squares commute by $\mathbb{k} G$-linearity of the decomposition $\mathbb{k}(G / H) \simeq M_{u} \oplus N_{u}$. Finally, the two lateral pieces commute by the above discussion. By Lemma 4.4, the morphism $w$ on the very right is retracted by $\underline{u}: \mathbb{k}(G / H) \rightarrow \mathbb{k}$. So, in order to check that $v\left(M_{u}\right)(g)$ is indeed our $u(g)$, it suffices to establish the following equality in $\mathbb{k}$ :

$$
{ }^{g} \operatorname{Res}_{H(g)}^{H}(\underline{u}) \circ(g \cdot) \circ \operatorname{Res}_{H(g)}^{H}(w)(1)=u(g) .
$$

Unfolding the definition of $w$ from (4.5), the above left-hand side becomes

$$
\underline{u}(g \cdot w(1))=\frac{1}{n} \sum_{d \in G / H} u(d)^{-1} u(g d) .
$$

Let us use a Mackey formula. Let $S \subset G$ be a set of representatives of $H(g) \backslash G / H$ and recall the Mackey bijection (3.6) of left $H(g)$-sets

$$
\coprod_{s \in S} H(g) / H(g, s) \stackrel{\sim}{\rightarrow} G / H
$$

given by $[x]_{H(g, s)} \mapsto[x s]_{H}$ where $H(g, s):=H(g) \cap{ }^{s} H=H \cap H^{g} \cap{ }^{s} H$. Using this change of variables $d=[x s]_{H}$ in the above sum, we get

$$
\begin{array}{rlrl}
\underline{u}(g \cdot w(1)) & =\frac{1}{n} \sum_{s \in S} \sum_{[x] \in H(g) / H(g, s)} u(s)^{-1} \cdot u(g x s) & & \text { since } x \in H \\
& =\frac{1}{n} \sum_{s \in S} u(s)^{-1} \cdot \sum_{[x] \in H(g) / H(g, s)} u(g s) & & \text { for } g x s={ }^{x} x g \text { and }{ }^{g} x \in H \\
& =\frac{1}{n} \sum_{s \in S} u(s)^{-1} \cdot[H(g): H(g, s)] \cdot u(g s) & \\
& =\frac{1}{n} \sum_{s \in S}[H(g): H(g, s)] \cdot u(g) & & \text { by Lemma 4.13 } \\
& =\frac{1}{n} \cdot|G / H| \cdot u(g)=u(g) . & &
\end{array}
$$

The penultimate equality uses again the same Mackey bijection (4.15).

4.16. Proposition. Let $M$ be an endotrivial $\mathbb{k} G$-module in $T(G, H)$. Suppose that $v(M)$ $=1$ in $A(G, H)$. Then $M \simeq \mathbb{k}$ in $\mathbb{k} G$-stab.

Proof. Let $\xi: \mathbb{k} \rightarrow \operatorname{Res}_{H}^{G} M$ be a $\mathbb{k} H$-linear homomorphism which is an isomorphism in $\mathbb{k} H$-stab. The assumption about $v(M)=1$ implies that for every $H$-secant element $g \in G$, the following diagram commutes in $\mathbb{k} H(g)$-stab:

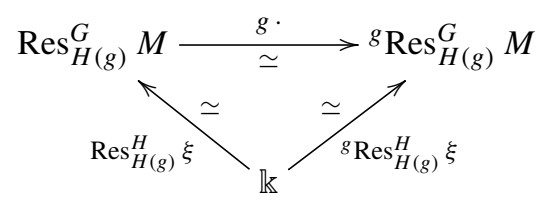


On the other hand, if $g$ is not $H$-secant, the same diagram trivially commutes in $\mathbb{k} H(g)$-stab $=0$. Therefore, diagram (4.17) commutes in $\mathbb{k} H(g)$-stab for all $g \in G$. Let us now define a $\mathbb{k} G$-linear homomorphism $\hat{\xi}: \mathbb{k} \rightarrow M$ by the composition

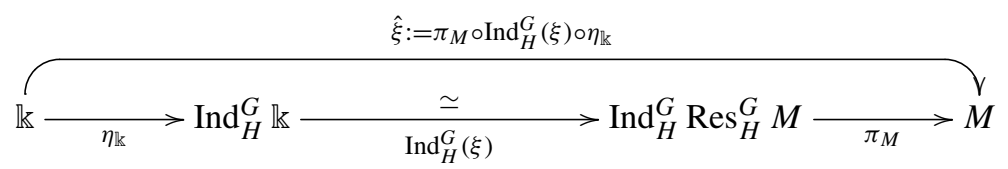

where $\eta:$ Id $\rightarrow$ Ind Res is the unit of the adjunction and $\pi:$ Ind Res $\rightarrow$ Id is the retraction of $\eta$ described in Remark 3.4. We claim that $\hat{\xi}$ is an isomorphism in $\mathbb{k} G$-stab. By Remark 3.4, it suffices to see that its restriction $\operatorname{Res}_{H}^{G}(\hat{\xi})$ is an isomorphism in $\mathbb{k} H$-stab. We claim more precisely that $\operatorname{Res}_{H}^{G}(\hat{\xi})=\xi$ in $\mathbb{k} H$-stab. This is not a mere property of the adjunction but will require (4.17) above. Applying $\operatorname{Res}_{H}^{G}$ to the last diagram, we get the upper part of the following commutative diagram in $\mathbb{k} H$-stab:

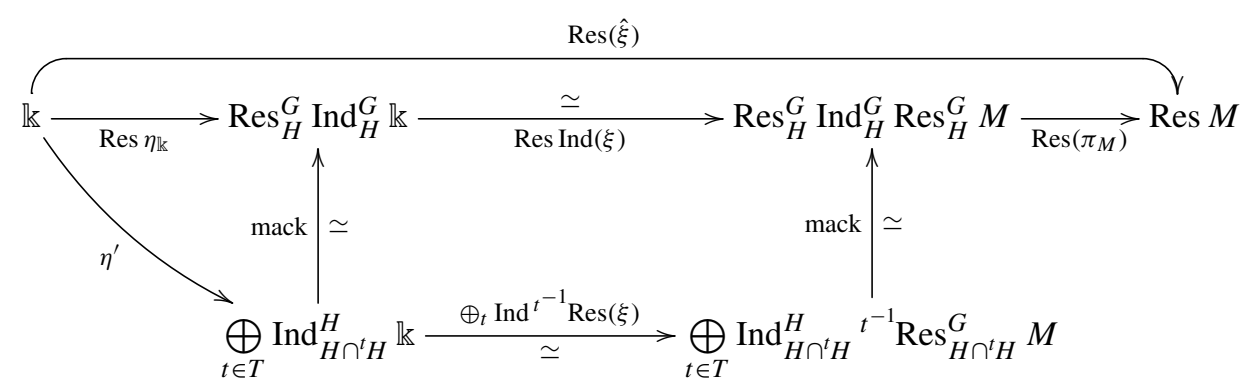

in which the lower part is constructed as follows. The vertical isomorphisms mack : $\bigoplus_{t \in T} \operatorname{Ind}_{H \cap{ }^{t} H}^{H}{ }^{t-1} \operatorname{Res}_{H \cap t}^{H} \rightarrow \operatorname{Res}_{H}^{G} \operatorname{Ind}_{H}^{G}$ are Mackey isomorphisms (3.7), for any choice of a set $T \subset G$ of representatives of $H \backslash G / H$. Explicitly, the component mack $: \mathbb{k} H \bigotimes_{\mathbb{k}\left(H \cap{ }^{t} H\right)} N \rightarrow \mathbb{k} G \bigotimes_{\mathbb{k} H} N$ is given by $\operatorname{mack}(x \otimes y)=(x t) \otimes y$. They are applied to the $\mathbb{k} H$-modules $N=\mathbb{k}$ and $N=\operatorname{Res}_{H}^{G} M$ respectively. Finally, $\eta^{\prime}: \mathbb{k} \rightarrow$ $\bigoplus_{t \in T} \operatorname{Ind}_{H \cap^{t} H}^{H} \mathbb{k}=\bigoplus_{t \in T} \mathbb{k} H \otimes_{\mathbb{k}\left(H \cap{ }^{t} H\right)} \mathbb{k}$ is defined by $1 \mapsto \sum_{t \in T} \sum_{x \in H /\left(H \cap \cap^{t} H\right)} x \otimes 1$, that is, $\eta^{\prime}=\operatorname{mack}^{-1} \circ \operatorname{Res}\left(\eta_{\mathbb{k}}\right)$. Hence the left-hand triangle commutes.

In each term of the bottom morphism, we can use (4.17) and replace every ${ }^{t^{-1}} \operatorname{Res}(\xi)$ by $\left(t^{-1} \cdot\right) \circ \operatorname{Res}(\xi)$, since that relation holds in $\mathbb{k}\left(H \cap{ }^{t} H\right)$-stab and can then be induced to $H$. Therefore, in $\mathbb{k} H$-stab, the morphism $\operatorname{Res}(\hat{\xi})$ is equal to

$$
\operatorname{Res}\left(\pi_{M}\right) \circ \operatorname{mack} \circ\left(\oplus_{t} \operatorname{Ind}\left(\left(t^{-1} \cdot\right) \circ \operatorname{Res}(\xi)\right)\right) \circ \eta^{\prime}
$$

We claim that the latter composition is simply $\xi$ in $\mathbb{k} H$-mod already. We compute the image of $1 \in \mathbb{k}$ under this morphism and get in $M$ the equalities 


$$
\begin{array}{rlr}
\operatorname{Res} & \left(\pi_{M}\right) \circ \operatorname{mack} \circ\left(\oplus_{t} \operatorname{Ind}\left(\left(t^{-1} \cdot\right) \circ \operatorname{Res}(\xi)\right)\right) \circ \eta^{\prime}(1) & \\
& =\frac{1}{n} \sum_{t \in T} \sum_{[x] \in H /\left(H \cap{ }^{t} H\right)} x t \cdot t^{-1} \cdot \xi(1) & \text { unfolding the definitions } \\
& =\frac{1}{n} \sum_{t \in T} \sum_{[x] \in H /\left(H \cap{ }^{t} H\right)} \xi(x \cdot 1) & \text { by } H \text {-linearity of } \xi \\
= & \frac{1}{n} \sum_{t \in T} \sum_{[x] \in H /\left(H \cap{ }^{t} H\right)} \xi(1) & \text { for the module } \mathbb{k} \text { is trivial } \\
= & \frac{1}{n}\left(\sum_{t \in T}\left|H /\left(H \cap{ }^{t} H\right)\right|\right) \cdot \xi(1)=\frac{|G / H|}{n} \xi(1)=\xi(1) . &
\end{array}
$$

The penultimate equality uses again the same Mackey bijection (3.6).

Everything is now in place to wrap it up:

Proof of Theorems 2.8 and 2.9. We have the well-defined maps $\alpha: A(G, H) \rightarrow T(G, P)$ of Proposition 4.6 and $v: T(G, P) \rightarrow A(G, H)$ of Proposition 4.12, where we also saw that $v$ is a group homomorphism. Proposition 4.14 tells us that $v \circ \alpha$ is the identity, hence $v$ is surjective. Proposition 4.16 shows that $v$ is injective.

4.18. Remark. It follows that $\alpha$ is a group homomorphism, which was not obvious from its definition.

We also have naturality with respect to restriction to subgroups:

4.19. Proposition. Let $H \leq G^{\prime} \leq G$ be an intermediate subgroup. Then every weak $H$-homomorphism from $G$ to $\mathbb{k}^{\times}$restricts to a weak $H$-homomorphism from $G^{\prime}$ to $\mathbb{k}^{\times}$. The induced homomorphism $A(G, H) \rightarrow A\left(G^{\prime}, H\right)$ is compatible with the restriction $\operatorname{Res}_{G^{\prime}}^{G}: T(G, H) \rightarrow T\left(G^{\prime}, H\right)$, via the isomorphisms $v$ and $\alpha$.

Proof. The properties that $p$ divides $\left|H \cap H^{g}\right|$ or $\left|H \cap H^{g_{1}} \cap H^{g_{2} g_{1}}\right|$ are unchanged if we consider elements $g, g_{1}, g_{2} \in G^{\prime}$ as elements of $G$. Consequently, the restriction of $u: G \rightarrow \mathbb{k}^{\times}$to $G^{\prime}$ will satisfy conditions (WH1-3) for $G^{\prime}$. So, $\operatorname{Res}_{G^{\prime}}^{G}: A(G, H) \rightarrow$ $A\left(G^{\prime}, H\right)$ is well-defined. It is easy to check that the following diagram commutes

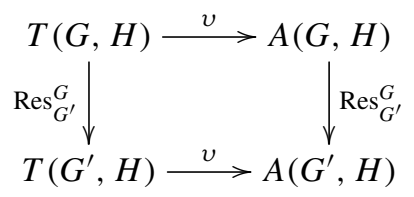

since we can use the same isomorphism $\xi: \mathbb{k} \stackrel{\sim}{\rightarrow} \operatorname{Res}_{H}^{G} M=\operatorname{Res}_{H}^{G^{\prime}} M^{\prime}$ in $\mathbb{k} H$-stab, for $M$ and for $M^{\prime}:=\operatorname{Res}_{G^{\prime}}^{G} M$. It follows that $\alpha=v^{-1}$ is also compatible with restriction, although this was maybe less obvious a priori.

Let us finish with the two extreme cases of Example 2.3. 
4.20. Example. Suppose that $H \triangleleft G$ is a normal subgroup. Then every element $g \in G$ is $H$-secant and we observed in Example 2.3(1) that $A(G, H) \cong \operatorname{Hom}\left(G / H, \mathbb{k}^{\times}\right)$. Given $\rho \in \operatorname{Hom}\left(G / H, \mathbb{k}^{\times}\right)$, let us write $\mathbb{k}_{\rho}$ for the one-dimensional representation $g \cdot x=$ $\rho([g]) x$ of $G$. It clearly belongs to $T(G, H)$. The associated weak $H$-homomorphism $u=v\left(\mathbb{k}_{\rho}\right)$ of Construction 2.5 is characterized by diagram (2.7) for $M=\mathbb{k}$ and $\xi=\mathrm{id}$, from which it follows that $u(g)=\rho(g)$. In other words, $v\left(\mathbb{k}_{\rho}\right)=\rho$, as one would of course expect. It also follows that $\alpha(\rho)=\mathbb{k}_{\rho}$, i.e. $M_{\rho}$ is isomorphic to $\mathbb{k}_{\rho}$ in the stable category $\mathbb{k} G$-stab. This last fact is less evident but can also be checked directly from the definition of $M_{\rho}$ with the idempotent $e_{\rho}$.

4.21. Example. Suppose that $H \leq G$ is strongly $p$-embedded, as in Example 2.3(2), where we saw that $A(G, H)=1$. Then $T(G, H)=1$. This is a well-known fact, which trivially follows from the underlying property that the restriction functor $\operatorname{Res}_{H}^{G}$ : $\mathbb{k} G$-stab $\stackrel{\sim}{\rightarrow} \mathbb{k} H$-stab is an equivalence of tensor categories in that case.

\section{Some corollaries}

The following corollary is known in case $O_{p}(G) \neq 1$, by [13, Lemma 2.6].

5.1. Corollary. Let $H \leq G$ be a subgroup of index prime to $p$. Suppose that $H$ contains some subgroup $K \triangleleft G$, normal in $G$ and of order divisible by $p$. Then the kernel $\operatorname{Ker}(T(G) \rightarrow T(H))$ consists only of one-dimensional representations, i.e. it is isomorphic to $\left\{\rho \in \operatorname{Hom}_{\mathrm{gps}}\left(G, \mathbb{k}^{\times}\right) \mid \rho(H)=1\right\}$.

Proof. For all $g_{1}, g_{2} \in G$, the subgroup $H \cap H^{g_{1}} \cap H^{g_{2} g_{1}}$ contains $K$, hence (WH3) holds. So every weak $H$-homomorphism $u: G \rightarrow \mathbb{k}^{\times}$is a group homomorphism.

5.2. Remark. It is well-known that the abelian group $T(G, H)$ is finite. Indeed, every $M \in T(G, H)$ appears as a direct summand of $\mathbb{k}(G / H)$ (see Theorem 2.9) and finiteness follows by the Krull-Schmidt Theorem. This gives an upper bound $|T(G, H)| \leq[G: H]$. So, $T(G, H)$ is a torsion abelian group and we can discuss the order of its elements. The following observation can be deduced from combining [13, Lemma 2.6] and [4, Prop. 2.6]. Our proof is direct.

5.3. Corollary. Let $H \leq G$ be a subgroup of index prime to $p$. Then there is no p-torsion in $\operatorname{Ker}(T(G) \rightarrow T(H))$. That is, if $M$ is a $\mathbb{k} G$-module such that $M^{\otimes p} \simeq \mathbb{k}$ in $\mathbb{k} G$-stab and $\operatorname{Res}_{H}^{G} M \simeq \mathbb{k}$ in $\mathbb{k} H$-stab then $M \simeq \mathbb{k}$ in $\mathbb{k} G$-stab already.

Proof. Let $u: G \rightarrow \mathbb{k}^{\times}$be a weak $H$-homomorphism such that $u^{p}=1$. Since the field $\mathbb{k}$ has characteristic $p$, the relation $u(g)^{p}=1$ forces $u(g)=1$ for all $g \in G$.

5.4. Remark. Actually, the orders of elements of $T(G, H)$ are related to the coefficient field $\mathbb{k}$. So, let us write $T_{\mathbb{k}}(G, H)$ and $A_{\mathbb{k}}(G, H)$ when we want to emphasize the choice of $\mathbb{k}$. As in Proposition 4.19, we can show that $v: T_{\mathbb{k}}(G, H) \rightarrow A_{\mathbb{k}}(G, H)$ and $\alpha$ : $A_{\mathbb{k}}(G, H) \rightarrow T_{\mathbb{k}}(G, H)$ are natural in $\mathbb{k}$.

Interestingly, for a $p$-group $P$, the group $T_{\mathbb{k}}(P)$ almost never depends on the field $\mathbb{k}$, except sometimes in characteristic 2, with quaternion groups. See [16, §2]. As we shall now see, the kernel $T_{\mathbb{k}}(G, P)$ does depend on $\mathbb{k}$, although in a nice way. 
5.5. Corollary. Let $H \leq G$ be a subgroup of index prime to $p$.

(a) For every field extension $\mathbb{k}^{\prime} / \mathbb{k}$, the homomorphism $T_{\mathbb{k}_{\mathrm{k}}}(G, H) \rightarrow T_{\mathbb{k}^{\prime}}(G, H)$ is injective. Moreover, an element $M \in T_{\mathbb{k}^{\prime}}(G, H)$ of order $d$ belongs to the image of this homomorphism (i.e. is defined over $\mathbb{k}$ ) if and only if $\mathbb{k}$ admits a primitive $d^{\text {th }}$ root of unity.

(b) Let $\mathbb{k}^{\prime} / \mathbb{k}$ be a field extension and suppose that for every $d \leq[G: H]$, every $d^{\text {th }}$ root of unity in $\mathbb{k}^{\prime}$ already belongs to $\mathbb{k}$, e.g. if $\mathbb{k}$ and $\mathbb{k}^{\prime}$ are both algebraically closed. Then $T_{\mathbb{k}}(G, H)=T_{\mathbb{R}^{\prime}}(G, H)$.

(c) Let $q$ be a power of $p$ and let $\mathbb{k}$ be a field containing the finite field $\mathbb{F}_{q}$. An element $M \in T_{\mathbb{k}_{\mathrm{k}}}(G, H)$ of order $d$ is defined over $\mathbb{F}_{q}$ if and only if $d$ divides $q-1$.

Proof. All these properties are easy to verify for $A_{\mathbb{k}}(G, H)$, hence can be transported to $T_{\mathrm{k}}(G, H)$. Indeed, a weak $H$-homomorphism $u \in A_{\mathrm{k}}(G, H)$ has order $d$ if and only if every $u(g)$ is a $d^{\text {th }}$ root of unity and some $u(g)$ is a primitive one. Details are left to the reader.

5.6. Example. Let $H \leq G$ be a subgroup of odd index and $\mathbb{F}_{2}$ the field with two elements. Then restriction $T_{\mathbb{F}_{2}}(G) \rightarrow T_{\mathbb{F}_{2}}(H)$ is injective. Indeed $\left(\mathbb{F}_{2}\right)^{\times}=1$.

Acknowledgments. I am thankful to Nadia Mazza, Serge Bouc, Jon Carlson and Jacques Thévenaz for numerous discussions, big or small, around the theme of this paper and to the referee for elegantly revamping Lemma 3.8 and the proof of Lemma 4.4.

This research was supported by NSF grant DMS-0969644.

\section{References}

[1] Alperin, J.: MathSciNet review of [16] MR 2336639

[2] Bouc, S.: The Dade group of a p-group. Invent. Math. 164, 189-231 (2006) Zbl 1099.20004 MR 2207787

[3] Carlson, J. F., Hemmer, D. J., Mazza, N.: The group of endotrivial modules for the symmetric and alternating groups. Proc. Edinburgh Math. Soc. (2) 53, 83-95 (2010) Zbl 1217.20004 MR 2579680

[4] Carlson, J. F., Mazza, N., Nakano, D. K.: Endotrivial modules for the symmetric and alternating groups. Proc. Edinburgh Math. Soc. (2) 52, 45-66 (2009) Zbl 1165.20005 MR 2475880

[5] Carlson, J. F., Mazza, N., Thévenaz, J.: Endotrivial modules for $p$-solvable groups. Trans. Amer. Math. Soc. 363, 4979-4996 (2011) Zbl 1250.20007 MR 2806698

[6] Carlson, J. F., Nakano, D. K.: Endotrivial modules for finite group schemes. J. Reine Angew. Math. 653, 149-178 (2011) Zbl 1226.20039 MR 2794629

[7] Carlson, J. F., Thévenaz, J.: The classification of endo-trivial modules. Invent. Math. 158, 389-411 (2004) Zbl 1110.20004 MR 2096798

[8] Carlson, J. F., Thévenaz, J.: The classification of torsion endo-trivial modules. Ann. of Math. (2) 162, 823-883 (2005) Zbl 1110.20005 MR 2183283

[9] Chouinard, L. G.: Projectivity and relative projectivity over group rings. J. Pure Appl. Algebra 7, 287-302 (1976) Zbl 0327.20020 MR 0401943

[10] Happel, D.: Triangulated Categories in the Representation Theory of Finite-Dimensional Algebras, London Math. Soc. Lecture Note Ser. 119, Cambridge Univ. Press, Cambridge (1988) Zbl 0635.16017 MR 0935124 
[11] Lassueur, C.: Relative projectivity and relative endotrivial modules. J. Algebra 337, 285-317 (2011) Zbl 1257.20003 MR 2796076

[12] Mazza, N.: The group of endotrivial modules in the normal case. J. Pure Appl. Algebra 209, 311-323 (2007) Zbl 1117.20009 MR 2293311

[13] Mazza, N., Thévenaz, J.: Endotrivial modules in the cyclic case. Arch. Math. (Basel) 89, 497-503 (2007) Zbl 1155.20009 MR 2371685

[14] Neeman, A.: Triangulated Categories. Ann. of Math. Stud. 148, Princeton Univ. Press (2001) Zbl 0974.18008 MR 1812507

[15] Robinson, G. R.: Endotrivial irreducible lattices. J. Algebra 335, 319-327 (2011) Zbl 1254.20004 MR 2792579

[16] Thévenaz, J.: Endo-permutation modules, a guided tour. In: Group Representation Theory, EPFL Press, Lausanne, 115-147 (2007) Zbl 1154.20004 MR 2336639 